\title{
Depression in doctors: A bitter pill to swallow
}

\author{
K Outhoff \\ Faculty of Health Sciences, University of Pretoria, South Africa \\ ${ }^{*}$ Corresponding author, email: kim.outhoff@up.ac.za
}

At some point in their career, at least a third of doctors suffer personal health problems such as injury, aging, or physical and mental illness, which detract from their ability to function properly and to practice medicine safely. In most, impairment is amenable to intervention, treatment, recovery or resolution. However, some conditions, notably depression, may be overrepresented in the medical profession, under-recognized, stigmatised and undertreated, which may have fatal consequences.

\section{Introduction}

Depression is a common mental disorder. The latest (2015) World Health Organization estimates revealed that over 320 million people, equivalent to $4.4 \%$ of the global population, suffer from depression. South Africans account for an estimated 2.4 million of these, which translates to $4.6 \%$ of the local population. Depression is more common in women $(5.1 \%)$ than in men (3.6\%), and the absolute number of people with depression is increasing, particularly in lower-income countries where populations are growing and aging. ${ }^{1}$ A meta-analysis of 1994-2014 depression studies showed an aggregate point prevalence of $12.9 \%$, a one-year prevalence of $7.2 \%$, and a lifetime prevalence of $10.8 \% .^{2}$ These discrepancies highlight that depression prevalence is difficult to assess accurately because of wide variations in diagnostic measures, and that studies using self-rated depression scales may produce significantly higher point prevalences of depression than clinician-rated.

Doctors appear to suffer from depression more than the general population and other professional groups. ${ }^{3}$ Recent meta-analyses of global studies estimate an overall prevalence of $27 \%$ in medical students, $29 \%$ in registrars and up to $60 \%$ in practising doctors. ${ }^{4}$ These figures differ widely between countries and specialties. For instance, a Cape Town study using a self-administered Beck's Depression Inventory revealed clinically relevant moderate to severe depression in $30 \%$ of public primary healthcare doctors, ${ }^{5}$ while an Australian study found that $18 \%$ of medical students and $21 \%$ of doctors had previously been diagnosed with depression. Medical students, younger doctors and women reported higher rates of psychological distress and mental health problems than men and older doctors. ${ }^{4}$ Meanwhile in America, there is some evidence that the prevalence of depression in doctors is similar to the general population. A cohort of male medical graduates from Johns Hopkins self-reported a lifetime prevalence of $12.8 \%$, similar to the $12 \%$ lifetime prevalence of major depression in American men, although their mean age of onset was significantly older. Self-identified lifetime prevalence of depression in American women doctors has been estimated at
$19.5 \%$, comparable with women in the general population and women professionals. It is thought that depression incidence across ethnic groups is similar, except for Asian women doctors, whose rates are lower. Importantly, the cross sectional and longitudinal prevalence of depression or depressive symptoms $(15-43.2 \%)$ is significantly higher in medical students and registrars/residents than in the general population, ${ }^{6,7}$ which appears to increase with calendar year of study. ${ }^{8}$

\section{Risk factors}

Although depression may affect anyone, the risk is increased by genetic predisposition, early traumatic life events, later bereavements, illnesses, relationship breakdowns, poverty, unemployment and problems caused by substance use. ${ }^{1}$ Stress as a precipitating factor is a common theme. ${ }^{9}$ (Table I)

Table I: Is depression a stress-induced organic disorder? ${ }^{9}$

Stress increases circulating pro-inflammatory cytokines, particularly TNFa and IL- 6 that activate microglia in the central nervous system leading to further pro-inflammatory cytokine release.

The immune cascade may ultimately reduce monoamine neurotransmitters, and in particular serotonin, as well as hippocampal neurogenesis.

Hypothalamic-pituitary-adrenal (HPA) axis dysregulation appears critical in the pathogenesis of depression.

Depressed people demonstrate increased peripheral cortisol levels and glucocorticoid receptor resistance, including in the hippocampus.

The cycle of elevated chronic neuro-inflammation and glucocorticoid functional resistance is accompanied by oxidative stress, as well as structural and functional changes in the brain, which may be restored with antidepressant treatment.

Doctors are exposed to more work-related stressors than many other professional groups, and are therefore vulnerable to depression. These include long hours, extensive workloads, the growing intensity and complexity of the job, relentless contact with patient ill health and emergencies, high levels of 
responsibility, rapid change within healthcare, institutional constraints such as discrimination and intimidation, lack of autonomy, low levels of support, loss of job satisfaction, low morale and the inability to attend to their personal lives. $4,7,10$ Causes of work stress may vary with medical discipline: family practitioners may face increased demands on primary care coupled with shrinking resources, ${ }^{7}$ while emergency care doctors and oncologists may suffer elements of posttraumatic stress. Furthermore, doctors facing complaints, often perceived as shameful catastrophic personal events, are at significantly increased risk of depression, anxiety and suicidal ideation. ${ }^{4}$

Personal attributes generally ensure patients are well cared for, yet also increase doctors' risks of developing mental health problems. Conscientiousness, obsessiveness and perfectionism may be more common in doctors, leading perhaps to rigidity, over-commitment, self-criticism, and an inability to unwind and replenish their inner resources. ${ }^{4}$ An inflated fear of making mistakes and a sense of diminished patient care may result in doctors experiencing overwhelming feelings of guilt, failure, shame and low self-esteem. ${ }^{3}$

Psychological defences that include depersonalisation and dissociation may make it difficult to create or maintain personal attachments or to recognise when the emotional burdens of work become unbearable. This may lead to spiralling distress, and increase the risks of depression and suicide. ${ }^{3}$ In fact, it has been asserted that more than $50 \%$ of US doctors experience burnout, a syndrome of emotional depletion and maladaptive detachment caused by prolonged occupational stress. ${ }^{11}$ This is particularly common in residents/registrars, and even more so in those pursuing surgical disciplines. ${ }^{11,12} \mathrm{~A}$ recent systematic review of 182 studies showed that overall physician burnout prevalence ranged from $0-80.5 \%$, figures highly dependent on rating scales and cut-off points for burnout, and limited by nosological debate. Emotional exhaustion, depersonalization, and low personal accomplishment ranged from 0-86\%, 0-90\%, and $0-87 \%$, respectively. ${ }^{13}$

A 2018 survey of 545 South African doctors revealed that over $40 \%$ either agree or strongly agree that they feel burnt out. Almost half cited heavier workloads, and the struggle to achieve an appropriate work/life balance, and more than a third experienced increasing stress and anxiety. An overwhelming majority (85\%) agreed or strongly agreed that patient expectations have increased in the last five years, and nearly $60 \%$ found it most challenging to manage unrealistic patient expectations in a climate of increasing litigation. ${ }^{14}$ The results of another 2018 study of 100 emergency doctors in Gauteng using the traditional Maslach's Burnout Inventory-Human Services Survey, indicated that a large proportion are at moderate to high risk of burnout. Not surprisingly, substantially higher emotional exhaustion levels were manifest in registrars (100\%) compared to medical officers and community service medical officers (86\%). ${ }^{15}$

Although the prevalence is difficult to establish (there are no standardised diagnostic criteria), burnout increases the risk of psychiatric conditions, including depression, anxiety, posttraumatic stress disorder, and suicidality. ${ }^{11}$ Importantly, there appears to be considerable overlap between symptoms of burnout and depression, ${ }^{16}$ a notion reinforced by an Austrian study that found that the Hamburg Burnout Inventory subscales correlated highly with the key features of depression (sadness, lack of interest, and diminished energy) rather than with each other, thus alluding to commonality between burnout and depression in doctors. ${ }^{13,17}$

Doctors have higher suicide rates, and depression is one of the major risk factors: ${ }^{6}$ Two decades ago, doctors' relative suicide risk compared to the general population was estimated at 1.1-3.4 for men and 2.5-5.7 for women. ${ }^{18}$ Compared to the general population, suicide is reportedly $40 \%$ higher in men and $130 \%$ higher in women doctors. ${ }^{12}$ Conflicting evidence suggests that suicide risk is greatest in psychiatrists and anaesthetists, community doctors and general practitioners. ${ }^{7}$ Besides mental distress, additional risk factors for suicide in doctors in particular include alcohol or other drug misuse and access to prescription medications. Drug overdose is the most common suicide method, and is used significantly more than in the general population. ${ }^{4}$ Suicidality may be reduced by better detection and adequate treatment of depression and other mental health problems. ${ }^{6}$

\section{Barriers to detection and effective treatment}

Depression is difficult to diagnose (Table II). In primary care, $30-50 \%$ of cases are reportedly missed, and screening may only be useful when a depressive disorder is suspected and in high-risk populations. ${ }^{19}$ Considering that approximately only $35 \%$ of doctors have a regular source of healthcare, it is not surprising that depression may be more under-recognised in this population compared to other professional groups. ${ }^{20}$ Selfdiagnosis is even more challenging.

Denial, stoicism and silence may result in delayed help-seeking and diagnosis. ${ }^{22}$ There may be a tacit expectation to be the "perfect" individual in the community, and from a doctor's perspective where illness is strongly perceived as (personal) failure, and health as success, the pressure to be invincible may be enormous. Mental distress may be misattributed to ineptitude or weakness, encouraging depressed doctors to conceal their illness from themselves and others. Self-judgement compromises access to care. Furthermore, diagnostic criteria include impairment of functioning, which may provide a getout clause for diagnosing depression. Although absenteeism occurs, presenteeism appears common in depressed doctors who are battling with issues surrounding a potential diagnosis of depression. ${ }^{7}$ Doctors may thus become the walking wounded as they soldier on while experiencing symptoms of depression such as feeling hopelessness, worthlessness, fatigue and indecision. ${ }^{10}$ Self-medicating with alcohol or recreational drugs may strengthen the illusion that all is well, but pose an additional risk factor for delayed care and suicide. A high prevalence of alcohol misuse (49\%), more than 5 times higher than in the general population (9\%), has been demonstrated in US surgery residents. ${ }^{23}$ 
Table II: Major Depressive Episode - Abridged DSM-V criteria ${ }^{19}$

A Five of the following features should be present most of the day, or nearly every day, for two weeks, representing a change of functioning (must include 1 or 2):

1. Depressed mood (feeling sad, empty, hopeless, tearful) nearly
every day, for most of the day
2. Marked loss of interest or pleasure in all or almost all activities
3. Significant weight loss or gain (more than $5 \%$ change in 1 month)
or an increase or decrease in appetite nearly every day
4. Insomnia or hypersomnia nearly every day
5. Observable psychomotor agitation or retardation
6. Fatigue or loss of energy nearly every day
7. Feelings of worthlessness or inappropriate or excessive guilt (not
merely self-reproach about being sick)
8. Diminished ability to think or concentrate, or indecisiveness, either
by subjective account or observed by others
9. Recurrent thoughts of death (not just fear of dying), suicidal
ideation, a suicide attempt, or a specific plan for committing
suicide

B The symptoms cause clinically significant distress or impairment in social, occupational or other important functioning.

C The symptoms are not due to a medical/organic factor or illness.

Episodes are classified as mild (few symptoms, mild functional impairment), moderate (minimum symptoms and functional impairment between mild and severe), severe (most symptoms, marked or greater functional impairment). ${ }^{19}$

(Adapted from American Psychiatric Association Diagnostic and Statistical Manual of Mental Disorders, Fifth Edition. May 2013)21

Failure to cope, coupled with the significant stigma that accompanies mental health problems, dissuades many from disclosing their depression. Doctors may fear naming, blaming and shaming, sanctions and job loss.7 An American survey of 2106 women doctors revealed that although almost 50\% believed they had met the criteria for mental illness, they had not sought treatment because they felt "they could manage independently, had limited time, were fearful of reporting it to a medical licensing board, and because they believed that a diagnosis was embarrassing or shameful,"24 supporting the view that fewer professions stigmatise mental health disorders more prominently than medicine. ${ }^{25}$ While some institutions offer free confidential professional mental health access, this may inadvertently be another potential source of stigmatisation. It has been suggested that registrars in particular, should all have a periodic mental health review, and rather than opting in, this should be an opting out service, in order to circumvent individual stigmatisation. ${ }^{22}$ Interestingly, many will admit to burnout, but not to depression. It has been suggested that the concept of burnout avoids pathologising workers' emotional responses to their jobs. ${ }^{13}$

Doctors are known to encounter discrimination in licensing, health insurance, and/or malpractice insurance. ${ }^{3}$ Lack of confidentiality and fear of documentation on their professional record may become additional barriers to accessing care. Administrators (and doctors) may forget that a diagnosis of depression does not necessarily mean impaired professional abilities. Fears of breaches of confidentiality as well as judgement may also compromise openness between depressed doctors and their potential treating healthcare professionals, further discouraging them from seeking help, or making a diagnosis more difficult. ${ }^{6}$ Depressed doctors thus face considerable personal (self, friends, family), professional and institutional stigma when trying to access care, potentially leaving them more isolated and vulnerable. ${ }^{26}$ Other barriers to accessing mental health services include a pessimistic view of their value, fear of taking psychotropic medication, lack of energy or motivation, and cost. ${ }^{3,22}$

\section{Treatment and recommendations}

When doctors do present for help, it may be difficult for the treating doctor/healthcare professional to see the patient, rather than a professional peer. Doctors may treat depressed doctors differently, engaging in medical talk and discussing academic papers or the latest research instead of their usual patient-doctor interaction. This social awkwardness may jeopardise effective treatment. Shared decision-making is discouraged initially, as the patient may benefit from taking a break from being a doctor and the agonies of self-diagnosis/treatment and impaired judgement until his/her health improves. ${ }^{3}$

Although depression may be a recurrent or chronic illness, full remission is the treatment goal. ${ }^{27}$ Therapeutic options include psychological and/or pharmacological, depending on disease severity. Psychological approaches include cognitive behaviour therapy, interpersonal psychotherapy and behavioural activation, which have strong evidence for efficacy in mild to moderate major depression, and as an adjunct to medication in severe depression. ${ }^{19}$

Antidepressants are recommended first-line treatments for moderate and severe major depression, irrespective of perceived cause, and for depression of any severity that has persisted for two or more years (persistent depressive disorder). Choice of antidepressant depends on individual and drug factors such as tolerability and relative safety in overdose. Selective serotonin reuptake inhibitors (SSRIs) and other newer antidepressants at full therapeutic dose are reasonable first line choices, whereas older tricyclic antidepressants and monoamine oxidase inhibitors should generally be reserved for treatment failure. ${ }^{19}$ According to guidelines, treatment should include scheduled follow-ups to monitor progress and enhance adherence, with access to a psychiatrist if there is a significant perceived risk of suicide, of harm to others or of severe self-neglect, if there are psychotic symptoms, a history or clinical suspicion of bipolar disorder, if insufficiently experienced to assess or manage a colleague's condition and if two or more attempts to treat a patient's depressive disorder with medication have failed, or resulted in insufficient response. ${ }^{19}$ These general measures improve outcomes.

Duration of antidepressant treatment at therapeutic dose is dictated by the risks of relapse. Where low, a minimum duration of 9-12 months after full remission should be considered, while two years or long-term treatment is advised in higher risk patients who have experienced two or more depressive episodes in the last few years. ${ }^{19}$ Cognitive behavioural therapy added to medication should be considered for patients with residual 
symptoms or at high risk of relapse, and mindfulness-based cognitive therapy added to usual treatment may be useful for preventing relapse in those with $\geq 3$ previous episodes. ${ }^{19}$

Importantly, socio-cultural factors precipitating or maintaining depression should be considered and addressed if possible. Occupational stress resilience strategies include mindfulness, ${ }^{28}$ making time for exercise/hobbies/holidays, spending quality time with partners/family/friends, limiting alcohol use, selfmonitoring, limit setting, and attitudes that promote constructive and healthy engagement with the challenges at work. ${ }^{29}$ Increased resources, collegial support, and a willingness to talk about vulnerability may enable doctors to feel less isolated and better able to cope with their emotionally demanding jobs. ${ }^{30}$ Greater dispositional mindfulness and trait resilience in US surgery residents have been associated with high-stress risk reduction of $85 \%$ and $65 \%$ respectively, as well as significant reductions in risks of burnout, depression, anxiety and suicidality. ${ }^{23}$ However, occupational stress and burnout require action not only at individual, but also at group, social and institutional levels..$^{31,32}$

In conclusion, doctors are vulnerable to depression. Yet they seek treatment less frequently and die by suicide more than the general population. ${ }^{33}$ This is a bitter pill to swallow. Entertaining and accepting a diagnosis of depression with equanimity and finding appropriate help, require considerable self-awareness and courage. ${ }^{34}$ The challenge for all doctors is to learn to recognize depression, not only in their patients but also in themselves and in their colleagues, and to transcend the numerous personal, professional and institutional barriers to effective treatment. ${ }^{27,35}$

\section{References}

1. World Health Organization: Depression and other common mental disorders: global health estimates. 2017.

2. Lim GY, Tam WW, Lu Y, Ho CS, Zhang MW, Ho RC. Prevalence of Depression in the Community from 30 Countries between 1994 and 2014. Scientific reports. 2018,8:2861.

3. Gerada C: Doctors, suicide and mental illness. BJPsych bulletin. 2018:1-4.

4. Bailey E, Robinson J, McGorry P: Depression and suicide among medical practitioners in Australia. Intern Med J. 2018;48:254-8.

5. Rossouw L, Seedat S, Emsley RA, Suliman S, Hagemeister D. The prevalence of burnout and depression in medical doctors working in the Cape Town Metropolitan Municipality community healthcare clinics and district hospitals of the Provincial Government of the Western Cape: a cross-sectional study. South African Family Practice. 2013;55:567-73.

6. Center $C$, Davis $M$, Detre $T$, et al. Confronting depression and suicide in physicians: A consensus statement. JAMA. 2003;289:3161-6.

7. Kinman $\mathrm{G}$, Teoh $\mathrm{K}$. What could make a difference to the mental health of UK doctors? A review of the research evidence. L Soc Occup Med. 2018. Available at: http://eprints.bbk.ac.uk/id/eprint/24540

8. Mata DA, Ramos MA, Bansal N, Khan R, Guille C, Di Angelantonio E, Sen S. Prevalence of depression and depressive symptoms among resident physicians: a systematic review and meta-analysis. JAMA. 2015;314:2373-83.

9. Yrondi A, Sporer M, Schmitt L, Arbus C. Major depressive disorder: An organic disorder! Elsevier. 2018.

10. Naidoo R. How GPs cope with Depression? Mental Health Matters. 2018:5:1-2.

11. Williford ML, Scarlet S, Meyers MO, Luckett DJ, Fine JP, Goettler CE, et al. Multipleinstitution comparison of resident and faculty perceptions of burnout and depression during surgical training. JAMA. Surgery. 2018;153(8):705-711
12. Hochberg MS, Berman RS, Kalet AL, Zabar SR, Gillespie C, Pachter HL. The stress of residency: recognizing the signs of depression and suicide in you and your fellow residents. Am J Surg. 2013;205:141-6.

13. Rotenstein $L S$, Torre $M$, Ramos $M A$, et al. Prevalence of burnout among physicians: A systematic review. JAMA. 2018;320:1131-50.

14. MPS News. Survey reveals over $40 \%$ of doctors feel burnt out 17 October 2018 [Accessed 20 November 2018]. Available at: https://www. medicalprotection.org/southafrica/for-members/news-centre/ news/2018/10/17/during-mental-health-awareness-month-medicalprotection-survey-reveals-over-40-of-doctors-feel-burnt-out?utm unique $=\&$ utm campaign $=2239266$ RSA\%20MED $\% 20$ Casebook $\% 20$ Connect\%20November\%202018\%20LS\&utm_medium=email\&utm . source $=$ MPS \&dm $i=2 F C 5,1 B Z T U, 46 Y D K P, 4 C 965,1$

15. Rajan $S$, Engelbrecht A. A cross-sectional survey of burnout amongst doctors in a cohort of public sector emergency centres in Gauteng, South Africa. Afr J Emerg Med. 2018;8(3)95-99.

16. Schonfeld IS, Bianchi R: Burnout and depression: two entities or one? J Clin Psychol. 2016;72:22-37.

17. Wurm W, Vogel K, Holl A, Ebner C, Bayer D, Mörkl S, et al. Depression-burnout overlap in physicians. PloS One. 2016;11:e0149913.

18. Lindeman $S$, Läärä $E$, Hakko $H$, Lönnqvist J. A systematic review on genderspecific suicide mortality in medical doctors. BJPsych. 1996;168:274-9.

19. Cleare A, Pariante CM, Young AH, Anderson IM, Christmas D, Cowen PJ, et al. Evidence-based guidelines for treating depressive disorders with antidepressants: a revision of the 2008 British Association for Psychopharmacology guidelines. J Psychopharmacol. 2015;29:459-525.

20. Gerada C. Doctors, suicide and mental disorders. BJPsych Bulletin 2018;42:165168. doi:10.1192/bjb.2018.11

21. Depressive Disorders. Diagnostic and Statistical Manual of Mental Disorders Fifth edition. [Accessed 11 Dec 2018]. Available at: https://dsm.psychiatryonline.org/ doi/10.1176/appi.books.9780890425596.dsm04\#

22. Batra M, McPhillips H, Shugerman R. Improving Resident Use of Mental Health Resources: It's Time for an Opt-Out Strategy to Address Physician Burnout and Depression (Commentary). J Grad Med Educ. 2018;10:67-9.

23. Lebares CC, Guvva EV, Ascher NL, O'Sullivan PS, Harris HW, Epel ES. Burnout and stress among US surgery residents: psychological distress and resilience. J Am Coll Surg. 2018;226:80-90.

24. Gold KJ, Andrew LB, Goldman EB, Schwenk TL. "I would never want to have a mental health diagnosis on my record": a survey of female physicians on mental health diagnosis, treatment, and reporting. Gen Hosp Psychiatry. 2016;43:51-7.

25. Cohen D, Winstanley S, Greene $G$. Understanding doctors' attitudes towards self-disclosure of mental ill health. J Occup Med. 2016;66:383-9.

26. Henderson M, Brooks SK, del Busso L, Chalder T, Harvey SB, Hotopf M, et al. Shame! Self-stigmatisation as an obstacle to sick doctors returning to work: a qualitative study. BMJ Open 2012;2:e001776.

27. Glass RM. Awareness about depression: Important for all physicians. JAMA. 2003;289:3169-70.

28. Kabat-Zinn J, Hanh TN. Full catastrophe living: Using the wisdom of your body and mind to face stress, pain, and illness. Delta. 2009.

29. Kumar S. Burnout and doctors: prevalence, prevention and intervention. Healthcare: Multidisciplinary Digital Publishing Institute; 2016. p. 37.

30. Riley R, Spiers J, Buszewicz M, Taylor AK, Thornton G, Chew-Graham CA. What are the sources of stress and distress for general practitioners working in England? A qualitative study. BMJ Open 2018;8:e017361.

31. da Costa B, Pinto I. Stress, Burnout and Coping in Health Professionals: A Literature Review. J Psychol Brain Stud. 2017;1.

32. Shanafelt TD, Noseworthy JH. Executive leadership and physician well-being: nine organizational strategies to promote engagement and reduce burnout. Mayo Clinic Proceedings: Elsevier; 2017. pp. 129-46.

33. Middleton JL. Today I'm grieving a physician suicide. Ann Fam Med. 2008;6:267-9.

34. Reuter H. The cost of not covering payment of treatment for Depressive Disorders. S Afr Fam Pract. 2018;60:49-

35. Zorbas J. Doctors in training: It's OK to not be OK. Australian Medicine. 2017;29:20. 